\title{
New Results on the (Super) Edge-Magic Deficiency of Chain Graphs
}

\author{
Ngurah Anak Agung Gede ${ }^{1}$ and Adiwijaya ${ }^{2}$ \\ ${ }^{1}$ Department of Civil Engineering, Universitas Merdeka Malang, Jl. Taman Agung No. 1, Malang 65146, Indonesia \\ ${ }^{2}$ School of Computing, Telkom University, Jl. Telekomunikasi No. 1, Bandung 40257, Indonesia \\ Correspondence should be addressed to Adiwijaya; kang.adiwijaya@gmail.com
}

Received 3 February 2017; Accepted 29 May 2017; Published 12 July 2017

Academic Editor: Dalibor Froncek

Copyright (C) 2017 Ngurah Anak Agung Gede and Adiwijaya. This is an open access article distributed under the Creative Commons Attribution License, which permits unrestricted use, distribution, and reproduction in any medium, provided the original work is properly cited.

\begin{abstract}
Let $G$ be a graph of order $v$ and size $e$. An edge-magic labeling of $G$ is a bijection $f: V(G) \cup E(G) \rightarrow\{1,2,3, \ldots, v+e\}$ such that $f(x)+f(x y)+f(y)$ is a constant for every edge $x y \in E(G)$. An edge-magic labeling $f$ of $G$ with $f(V(G))=\{1,2,3, \ldots, v\}$ is called a super edge-magic labeling. Furthermore, the edge-magic deficiency of a graph $G, \mu(G)$, is defined as the smallest nonnegative integer $n$ such that $G \cup n K_{1}$ has an edge-magic labeling. Similarly, the super edge-magic deficiency of a graph $G, \mu_{s}(G)$, is either the smallest nonnegative integer $n$ such that $G \cup n K_{1}$ has a super edge-magic labeling or $+\infty$ if there exists no such integer $n$. In this paper, we investigate the (super) edge-magic deficiency of chain graphs. Referring to these, we propose some open problems.
\end{abstract}

\section{Introduction}

Let $G$ be a finite and simple graph, where $V(G)$ and $E(G)$ are its vertex set and edge set, respectively. Let $v=|V(G)|$ and $e=|E(G)|$ be the number of the vertices and edges, respectively. In [1], Kotzig and Rosa introduced the concepts of edge-magic labeling and edge-magic graph as follows: an edge-magic labeling of a graph $G$ is a bijection $f: V(G) \cup$ $E(G) \rightarrow\{1,2,3, \ldots, v+e\}$ such that $f(x)+f(x y)+f(y)$ is a constant, called the magic constant of $f$, for every edge $x y$ of $G$. A graph that admits an edge-magic labeling is called an edge-magic graph. A super edge-magic labeling of a graph $G$ is an edge-magic labeling $f$ of $G$ with the extra property that $f(V(G))=\{1,2,3, \ldots, e\}$. A super edge-magic graph is a graph that admits a super edge-magic labeling. These concepts were introduced by Enomoto et al. [2] in 1998.

In [1], Kotzig and Rosa introduced the concept of edgemagic deficiency of a graph. They define the edge-magic deficiency of a graph $G, \mu(G)$, as the smallest nonnegative integer $n$ such that $G \cup n K_{1}$ is an edge-magic graph. Motivated by Kotzig and Rosa's concept of edge-magic deficiency, Figueroa-Centeno et al. [3] introduced the concept of super edge-magic deficiency of a graph. The super edge-magic deficiency of a graph $G, \mu_{s}(G)$, is defined as the smallest nonnegative integer $n$ such that $G \cup n K_{1}$ is a super edge-magic graph or $+\infty$ if there exists no such $n$.

A chain graph is a graph with blocks $B_{1}, B_{2}, \ldots, B_{k}$ such that, for every $i, B_{i}$ and $B_{i+1}$ have a common vertex in such a way that the block-cut-vertex graph is a path. We will denote the chain graph with $k$ blocks $B_{1}, B_{2}, \ldots, B_{k}$ by $C\left[B_{1}, B_{2}, \ldots, B_{k}\right]$. If $B_{1}=\cdots=B_{t}=B$, we will write $C\left[B_{1}, B_{2}, \ldots, B_{k}\right]$ as $C\left[B^{(t)}, B_{t+1}, \ldots, B_{k}\right]$. If, for every $i, B_{i}=H$ for a given graph $H$, then $C\left[B_{1}, B_{2}, \ldots, B_{k}\right]$ is denoted by $k H$-path. Suppose that $c_{1}, c_{2}, \ldots, c_{k-1}$ are the consecutive cut vertices of $C\left[B_{1}, B_{2}, \ldots, B_{k}\right]$. The string of $C\left[B_{1}, B_{2}, \ldots, B_{k}\right]$ is $(k-2)$-tuple $\left(d_{1}, d_{2}, \ldots, d_{k-2}\right)$, where $d_{i}$ is the distance between $c_{i}$ and $c_{i+1}, 1 \leq i \leq k-2$. We will write $\left(d_{1}, d_{2}, \ldots, d_{k-2}\right)$ as $\left(d^{(t)}, d_{t+1}, \ldots, d_{k-2}\right)$, if $d_{1}=\cdots=d_{t}=d$.

For any integer $m \geq 2$, let $L_{m}=P_{m} \times P_{2}$. Let $\mathrm{TL}_{m}$ and $\mathrm{DL}_{m}$ be the graphs obtained from the ladder $L_{m}$ by adding a single diagonal and two diagonals in each rectangle of $L_{m}$, respectively. Thus, $\left|V\left(\mathrm{TL}_{m}\right)\right|=\left|V\left(\mathrm{DL}_{m}\right)\right|=2 m,\left|E\left(\mathrm{TL}_{m}\right)\right|=$ $4 m-3$, and $\left|E\left(\mathrm{DL}_{m}\right)\right|=5 m-4 . \mathrm{TL}_{m}$ and $\mathrm{DL}_{m}$ are called triangle ladder and diagonal ladder, respectively.

Recently, the author studied the (super) edge-magic deficiency of $k \mathrm{DL}_{m}$-path, $C\left[K_{4}^{(k)}, \mathrm{DL}_{m}, K_{4}^{(n)}\right]$, and $k C_{4}$-path with some strings. Other results on the (super) edge-magic 
deficiency of chain graphs can be seen in [4]. The latest developments in this area can be found in the survey of graph labelings by Gallian [5]. In this paper, we further investigate the (super) edge-magic deficiency of chain graphs whose blocks are combination of $\mathrm{TL}_{m}$ and $\mathrm{DL}_{m}$ and $K_{4}$ and $\mathrm{TL}_{m}$, as well as the combination of $C_{4}$ and $L_{m}$. Additionally, we propose some open problems related to the (super) edgemagic deficiency of these graphs. To present our results, we use the following lemmas.

Lemma 1 (see [6]). A graph $G$ is a super edge-magic graph if and only if there exists a bijective function $f: V(G) \rightarrow$ $\{1,2, \ldots, v\}$ such that the set $S=\{f(x)+f(y): x y \in E(G)\}$ consists of e consecutive integers.

Lemma 2 (see [2]). If $G$ is a super edge-magic graph, then $e \leq$ $2 v-3$.

\section{Main Results}

For $k \geq 3$, let $G=C\left[B_{1}, B_{2}, \ldots, B_{k}\right]$, where $B_{j}=\mathrm{TL}_{m}$ when $j$ is odd and $B_{j}=\mathrm{DL}_{m}$ when $j$ is even. Thus $G$ is a chain graph with $|V(G)|=(2 m-1) k+1$ and $|E(G)|=(1 / 2)(k+1)(4 m-3)+$ $(1 / 2)(k-1)(5 m-4)$ when $k$ is odd, or $|E(G)|=(k / 2)(4 m-3)+$ $(k / 2)(5 m-4)$ when $k$ is even. By Lemma 2 , it can be checked that $G$ is not super edge-magic when $m \geq 3$ and $k$ is even and when $m \geq 4$ and $k$ is odd. As we can see later, when $m=$ 3 and $k$ is odd, $G$ is super edge-magic. Next, we investigate the super edge-magic deficiency of $G$. Our first result gives its lower bound. This result is a direct consequence of Lemma 2, so we state the result without proof.

Lemma 3. Let $k \geq 3$ be an integer. For any integer $m \geq 3$,

$$
\begin{aligned}
& \mu_{s}(G) \\
& \geq \begin{cases}\left\lfloor\frac{1}{4} k(m-3)\right\rfloor+1, & \text { if } k \text { is even, } \\
\left\lfloor\frac{1}{4}(k(m-3)-(m-1))\right\rfloor+1, & \text { if } k \text { is odd. }\end{cases}
\end{aligned}
$$

Notice that the lower bound presented in Lemma 3 is sharp. We found that when $m$ is odd, the chain graph $G$ with particular string has the super edge-magic deficiency equal to its lower bound as we state in Theorem 4 . First, we define vertex and edge sets of $B_{j}$ as follows.

$V\left(B_{j}\right)=\left\{u_{j}^{i}, v_{j}^{i}: 1 \leq i \leq m\right\}$, for $1 \leq j \leq k . E\left(B_{j}\right)=$ $\left\{u_{j}^{i} u_{j}^{i+1}, v_{j}^{i} v_{j}^{i+1}: 1 \leq i \leq m-1\right\} \cup\left\{e_{j}^{i}\right.$ : where $e_{j}^{i}$ is either $u_{j}^{i} v_{j}^{i+1}$ or $\left.v_{j}^{i} u_{j}^{i+1}, 1 \leq i \leq m-1\right\} \cup\left\{u_{j}^{i} v_{j}^{i}: 1 \leq i \leq m\right\}$, for $1 \leq j \leq$ $k$, when $j$ is odd, and $E\left(B_{j}\right)=\left\{u_{j}^{i} u_{j}^{i+1}, v_{j}^{i} v_{j}^{i+1}, u_{j}^{i} v_{j}^{i+1}, v_{j}^{i} u_{j}^{i+1}\right.$ : $1 \leq i \leq m-1\} \cup\left\{u_{j}^{i} v_{j}^{i}: 1 \leq i \leq m\right\}$, for $1 \leq j \leq k$, when $j$ is even.

Theorem 4. Let $k \geq 3$ be an integer and $G=C\left[B_{1}, B_{2}, \ldots, B_{k}\right]$ with string $\left(m-1, d_{1}, m-1, d_{2}, m-1, \ldots, d_{(1 / 2)(k-3)}, m-1\right)$ when $k$ is odd or $\left(m-1, d_{1}, m-1, d_{2}, \ldots, m-1, d_{(1 / 2)(k-2)}\right)$ when $k$ is even, where $d_{1}, d_{2}, \ldots, d_{\lfloor(1 / 2)(k-2)\rfloor} \in\{m-1, m\}$. For any odd integer $m \geq 3$,

$$
\mu_{s}(G)= \begin{cases}\frac{1}{4} k(m-3)+1, & \text { if } k \text { is even, } \\ \frac{1}{4}(k-1)(m-3), & \text { if } k \text { is odd. }\end{cases}
$$

Proof. First, we define $G$ as a graph with vertex set $V(G)=$ $\bigcup_{j=1}^{k} V\left(B_{j}\right)$, where $u_{j}^{m}=v_{j+1}^{1}, 1 \leq j \leq k-1$, and edge set $E(G)=\bigcup_{j=1}^{k} E\left(B_{j}\right)$. Under this definition, $u_{j}^{m}=v_{j+1}^{1}, 1 \leq j \leq$ $k-1$, are the cut vertices of $G$.

Next, for $1 \leq i \leq m$ and $1 \leq j \leq k$, define the labeling $f: V(G) \cup \alpha K_{1} \rightarrow\{1,2,3, \ldots,(2 m-1) k+1+\alpha\}$, where $\alpha=(1 / 4) k(m-3)+1$ when $k$ is even or $\alpha=(1 / 4)(k-1)(m-3)$ when $k$ is odd, as follows:

$$
\begin{aligned}
& f(x) \\
& = \begin{cases}\frac{1}{4}(j-1)(9 m-7)+2 i-1, & \text { if } x=u_{j}^{i}, j \text { is odd, } \\
\frac{1}{4}(j-1)(9 m-7)+2 i, & \text { if } x=v_{j}^{i}, j \text { is odd, } \\
\beta+\frac{1}{2}(5 i-3), & \text { if } x=u_{j}^{i}, i \text { is odd, } j \text { is even, } \\
\beta+\frac{1}{2}(5 i-4), & \text { if } x=u_{j}^{i}, i \text { is even, } j \text { is even, } \\
\beta+\frac{1}{2}(5 i-7), & \text { if } x=v_{j}^{i}, i \text { is odd, } j \text { is even, } \\
\beta+\frac{1}{2}(5 i-6), & \text { if } x=v_{j}^{i}, i \text { is even, } j \text { is even, }\end{cases}
\end{aligned}
$$

where $\beta=(1 / 4)(j-2)(9 m-7)+2 m$.

Under the vertex labeling $f$, it can be checked that no labels are repeated, $f\left(u_{j}^{m}\right)=f\left(v_{j+1}^{1}\right), 1 \leq j \leq k-1$, $\{f(x)+f(y): x y \in E(G)\}$ is a set of $|E(G)|$ consecutive integers, and the largest vertex label used is $(1 / 4)(k-2)(9 m-$ $7)+(1 / 2)(9 m-3)$ when $k$ is even or $(1 / 4)(k-1)(9 m-7)+2 m$ when $k$ is odd. Also, it can be checked that $f\left(u_{j}^{i}\right)+f\left(v_{j}^{i+1}\right)=$ $f\left(v_{j}^{i}\right)+f\left(u_{j}^{i+1}\right)$ when $j$ is odd.

Next, label the isolated vertices in the following way.

Case $k$ Is Odd. In this case, we denote the isolated vertices with $\left\{z_{2 j-1}^{l} \mid 1 \leq l \leq(1 / 2)(m-3), 1 \leq j \leq(1 / 2)(k-1)\right\}$ and set $f\left(z_{2 j-1}^{l}\right)=f\left(v_{2 j-1}^{m}\right)+5 l$.

Case $k$ Is Even. In this case, we denote the isolated vertices with $\left\{z_{2 j-1}^{l} \mid 1 \leq l \leq(1 / 2)(m-3), 1 \leq j \leq k / 2\right\} \cup\left\{z_{0}\right\}$ and set $f\left(z_{2 j-1}^{l}\right)=f\left(v_{2 j-1}^{m}\right)+5 l$ and $f\left(z_{0}\right)=f\left(v_{k}^{m}\right)+1$.

By Lemma 1, $f$ can be extended to a super edge-magic labeling of $G \cup \alpha K_{1}$ with the magic constant $(k / 4)(27 m-21)+5$ when $k$ is even or $(1 / 4)(k-1)(27 m-21)+6 m$ when $k$ is odd. Based on these facts and Lemma 3, we have the desired result.

An example of the labeling defined in the proof of Theorem 4 is shown in Figure 1(a).

Notice that when $m=3$ and $k$ is odd, $\mu_{s}(G)=0$. In other words, the chain graph $G$ with string $\left(2, d_{1}, 2, d_{2}\right.$, $\left.2, \ldots, d_{(1 / 2)(k-3)}, 2\right)$, where $d_{i} \in\{2,3\}$, is super edge-magic 


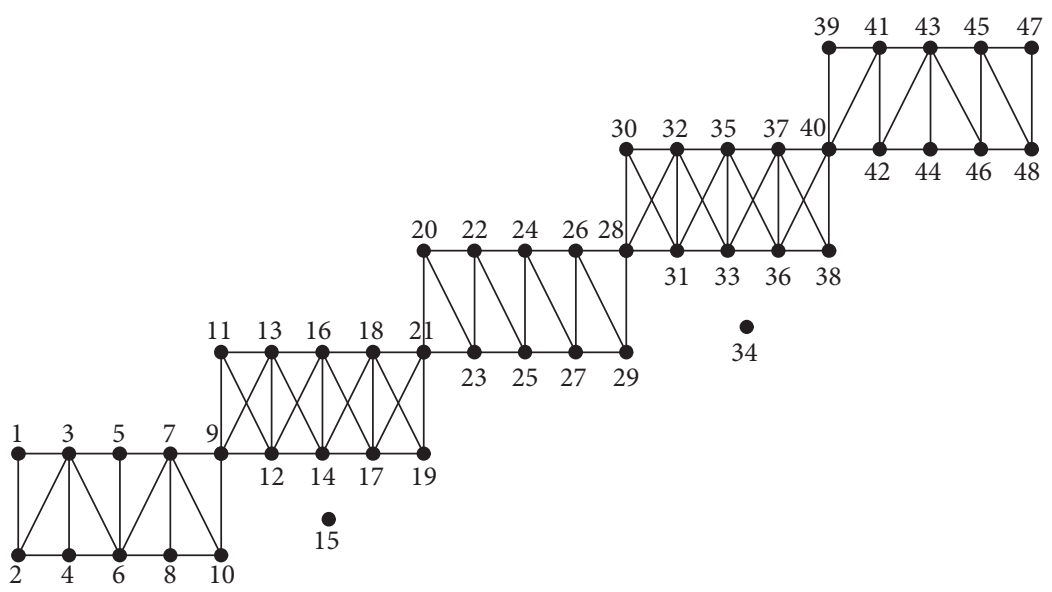

(a)

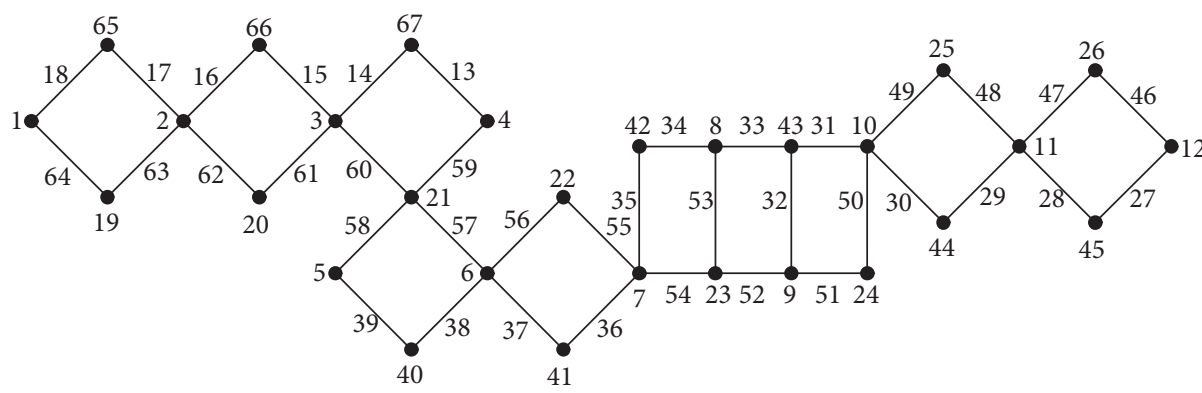

(b)

Figure 1: (a) Vertex labeling of $C\left[\mathrm{TL}_{5}, \mathrm{DL}_{5}, \mathrm{TL}_{5}, \mathrm{DL}_{5}, \mathrm{TL}_{5}\right] \cup 2 K_{1}$ with string $(4,5,4)$. (b) Vertex and edge labelings of $c\left[C_{4}^{(3+2)}, L_{4}, C_{4}^{(2)}\right]$ with string $\left(2,1^{(2)}, 2,4,2\right)$.

when $m=3$ and $k$ is odd. Based on this fact and previous results, we propose the following open problems.

Open Problem 1. Let $k \geq 3$ be an integer. For $m=2$, decide if there exists a super edge-magic labeling of $G$. Further, for any even integer $m \geq 2$, find the super edge-magic deficiency of G.

Next, we investigate the super edge-magic deficiency of the chain graph $H=C\left[K_{4}^{(p)}, \mathrm{TL}_{m}, K_{4}^{(q)}\right]$ with string $\left(1^{(p-1)}, d, 1^{(q-1)}\right)$, where $d \in\{m-1, m\} . H$ is a graph of order $3(p+q)+2 m$ and size $6(p+q)+4 m-3$. We define the vertex and edge sets of $H$ as follows: $V(H)=\left\{a_{i}, b_{i}: 1 \leq i \leq\right.$ $p\} \cup\left\{c_{i}: 1 \leq i \leq p+1\right\} \cup\left\{u_{j}, v_{j}: 1 \leq j \leq m\right\} \cup\left\{x_{t}, y_{t}: 1 \leq\right.$ $t \leq q\} \cup\left\{z_{t}: 1 \leq t \leq q+1\right\}$, where $c_{p+1}=u_{1}$ and $v_{m}=z_{1}$, and $E(H)=\left\{a_{i} b_{i}, a_{i} c_{i}, a_{i} c_{i+1}, b_{i} c_{i}, b_{i} c_{i+1}, c_{i} c_{i+1}: 1 \leq\right.$ $i \leq p\} \cup\left\{u_{j} v_{j} \mid 1 \leq j \leq m\right\} \cup\left\{u_{j} u_{j+1}, v_{j} v_{j+1}: 1 \leq j \leq\right.$ $m-1\} \cup\left\{e_{j}: e_{j}\right.$ is either $u_{j} v_{j+1}$ or $\left.v_{j} u_{j+1}, 1 \leq j \leq m-1\right\} \cup$ $\left\{x_{t} y_{t}, x_{t} z_{t}, x_{t} z_{t+1}, y_{t} z_{t}, y_{t} z_{t+1}, z_{t} z_{t+1}: 1 \leq t \leq q\right\}$. Hence, the cut vertices of $H$ are $c_{i}, 2 \leq i \leq p+1$, and $z_{t}, 1 \leq t \leq q$. Notice that $H$ has string $\left(1^{(p-1)}, m-1,1^{(q-1)}\right)$, if at least one of $e_{j}$ is $u_{j} v_{j+1}$, and its string is $\left(1^{(p-1)}, m, 1^{(q-1)}\right)$, if $e_{j}=v_{j} u_{j+i}$ for every $1 \leq j \leq m-1$.

Theorem 5. For any integers $p, q \geq 1$ and $m \geq 2, \mu_{s}(H)=0$.
Proof. Define a bijective function $g: V(H) \rightarrow$ $\{1,2,3, \ldots, 3(p+q)+2 m\}$ as follows:

$g(x)$

$$
= \begin{cases}3 i-2, & \text { if } x=a_{i}, 1 \leq i \leq p, \\ 3 i, & \text { if } x=b_{i}, 1 \leq i \leq p, \\ 3 i-1, & \text { if } x=c_{i}, 1 \leq i \leq p+1, \\ 3 p+2 j, & \text { if } x=u_{j}, 1 \leq j \leq m, \\ 3 p+2 j-1, & \text { if } x=v_{j}, 1 \leq j \leq m, \\ 3 p+2 m+3 t-2, & \text { if } x=x_{t}, 1 \leq t \leq q, \\ 3 p+2 m+3 t, & \text { if } x=y_{t}, 1 \leq t \leq q, \\ 3 p+2 m+3 t-4, & \text { if } x=z_{t}, 1 \leq t \leq q+1 .\end{cases}
$$

Under the labeling $g$, it can be checked that $g\left(c_{p+1}\right)=$ $g\left(u_{1}\right)$ and $g\left(v_{m}\right)=g\left(z_{1}\right)$. Also, it can be checked that $g\left(u_{j}\right)+$ $g\left(v_{j+1}\right)=g\left(v_{j}\right)+g\left(u_{j+1}\right), 1 \leq j \leq m-1$, and $\{g(x)+g(y) \mid$ $x y \in E(H)\}=\{3,4,5, \ldots, 6(p+q)+4 m-1\}$. By Lemma $1, g$ can be extended to a super edge-magic labeling of $H$ with the magic constant $9(p+q)+6 m$. Hence, $\mu_{s}(H)=0$.

Open Problem 2. For any integers $p, q \geq 1$ and $m \geq 2$, find the super edge-magic deficiency of $C\left[K_{4}^{(p)}, \mathrm{TL}_{m}, K_{4}^{(q)}\right]$ with string $\left(1^{(p-1)}, d, 1^{(q-1)}\right)$, where $d \in\{1,2,3, \ldots, m-2\}$. 
Next, we study the edge-magic deficiency of ladder $L_{m}$ and chain graphs whose blocks are combination of $C_{4}$ and $L_{m}$ with some strings. In [6], Figueroa-Centeno et al. proved that the ladder $L_{m}$ is super edge-magic for any odd $m$ and suspected that $L_{m}$ is super edge-magic for any even $m>2$. Here, we can prove that $L_{m}$ is edge-magic for any $m \geq 2$ by showing its edge-magic deficiency is zero. The result is presented in Theorem 6 .

Theorem 6. For any integer $m \geq 2, \mu\left(L_{m}\right)=0$.

Proof. Let $V\left(L_{m}\right)=\left\{u_{i}, v_{i}: 1 \leq i \leq m\right\}$ and $E(G)=$ $\left\{u_{i} u_{i+1}, v_{i} v_{i+1}: 1 \leq i \leq m-1\right\} \cup\left\{u_{i} v_{i}: 1 \leq i \leq m\right\}$ be the vertex set and edge set, respectively, of $L_{m}$. It is easy to verify that the labeling $h: V\left(L_{m}\right) \cup E\left(L_{m}\right) \rightarrow\{1,2,3, \ldots, 5 m-2\}$ is a bijection and, for every $x y \in E\left(L_{m}\right), h(x)+h(x y)+h(y)=6 m$.

$$
h(x)= \begin{cases}i, & \text { if } x=u_{i}, i \text { is odd, } \\ 3 m+\frac{1}{2}(i-2), & \text { if } x=u_{i}, i \text { is even, } \\ m+\frac{1}{2}(i+1), & \text { if } x=v_{i}, i \text { is odd, } \\ i, & \text { if } x=v_{i}, i \text { is even, } \\ 3 m-\frac{1}{2}(3 i-1), & \text { if } x=u_{i} u_{i+1}, \quad i \text { is odd, } \\ 3 m-\frac{3}{2} i, & \text { if } x=u_{i} u_{i+1}, \quad i \text { is even, } \\ 5 m-\frac{3}{2}(i+1), & \text { if } x=v_{i} v_{i+1}, \quad i \text { is odd, } \\ 5 m-\frac{1}{2}(3 i+2), & \text { if } x=v_{i} v_{i+1}, \quad i \text { is even, } \\ 5 m-\frac{1}{2}(3 i+1), & \text { if } x=u_{i} v_{i}, i \text { is odd, } \\ 3 m-\frac{1}{2}(3 i-2), & \text { if } x=u_{i} v_{i}, i \text { is even. }\end{cases}
$$

Thus, $\mu\left(L_{m}\right)=0$ for every $m \geq 2$.

Theorem 7. Let $p$ and $q \geq 1$ be integers.

(a) If $m \geq 2$ is an even integer and $F_{1}=C\left[C_{4}^{(p)}, L_{m}, c_{4}^{(q)}\right]$ with string $\left(2^{(p-1)}, m, 2^{(q-1)}\right)$, then $\mu\left(F_{1}\right)=0$.

(b) If $m \geq 3$ is an odd integer and $F_{2}=C\left[C_{4}^{(p)}, L_{m}, c_{4}^{(q)}\right]$ with string $\left(2^{(p-1)}, m-1,2^{(q-1)}\right)$, then $\mu\left(F_{2}\right)=0$.

Proof. (a) First, we introduce a constant $\lambda$ as follows: $\lambda=1$, if $m$ is odd and $\lambda=2$, if $m$ is even. Next, we define $F_{1}$ as a graph with $V\left(F_{1}\right)=\left\{a_{i}, b_{i}: 1 \leq i \leq p\right\} \cup\left\{c_{i}: 1 \leq i \leq p+1\right\} \cup\left\{u_{j}, v_{j}: 1 \leq\right.$ $j \leq m\} \cup\left\{x_{t}, y_{t}: 1 \leq t \leq q\right\} \cup\left\{z_{t}: 1 \leq t \leq q+1\right\}$, where $c_{p+1}=v_{1}$ and $u_{m}=z_{1}$, and $E(H)=\left\{c_{i} a_{i}, c_{i} b_{i}, a_{i} c_{i+1}, b_{i} c_{i+1}: 1 \leq\right.$ $i \leq p\} \cup\left\{u_{j} v_{j} \mid 1 \leq j \leq m\right\} \cup\left\{u_{j} u_{j+1}, v_{j} v_{j+1}: 1 \leq j \leq\right.$ $m-1\} \cup\left\{z_{t} x_{t}, z_{t} y_{t}, x_{t} z_{t+1}, y_{t} z_{t+1}: 1 \leq t \leq q\right\}$. The cut vertices of $F_{1}$ are $c_{i}, 2 \leq i \leq p+1$, and $z_{t}, 1 \leq t \leq q$.

Next, define a bijection $f_{1}: V\left(F_{1}\right) \cup E\left(F_{1}\right) \rightarrow\{1,2,3, \ldots$, $7(p+q)+5 m-2\}$ as follows:

$$
\begin{aligned}
& f_{1}(x) \\
& \begin{cases}4(p+q)+3 m+i-1, & \text { if } x=a_{i}, 1 \leq i \leq p, \\
p+q+m+i, & \text { if } x=b_{i}, 1 \leq i \leq p, \\
i, & \text { if } x=c_{i}, 1 \leq i \leq p+1, \\
5 p+4 q+3 m+\frac{1}{2}(j-1), & \text { if } x=u_{j}, j \text { is odd, } \\
p+j, & \text { if } x=u_{j}, j \text { is even, } \\
p+j, & \text { if } x=v_{j}, j \text { is odd, } \\
2 p+q+m+\frac{j}{2}, & \text { if } x=v_{j}, j \text { is even, } \\
5 p+4 q+\gamma_{1}+t, & \text { if } x=x_{t}, 1 \leq t \leq q, \\
2 p+q+\gamma_{2}+t, & \text { if } x=y_{t}, 1 \leq t \leq q, \\
p+m+t-1, & \text { if } x=z_{t}, 1 \leq t \leq q+1, \\
4(p+q)+3 m+1-2 i, & \text { if } x=c_{i} a_{i}, 1 \leq i \leq p, \\
7(p+q)+5 m-2 i, & \text { if } x=c_{i} b_{i}, 1 \leq i \leq p, \\
4(p+q)+3 m-2 i, & \text { if } x=a_{i} c_{i+1}, 1 \leq i \leq p, \\
7(p+q)+5 m-1-2 i, & \text { if } x=b_{i} c_{i+1}, 1 \leq i \leq p, \\
2 p+4 q+3 m-\frac{1}{2}(3 j+1), & \text { if } x=u_{j} u_{j+1}, j \text { is odd, } \\
2 p+4 q+3 m-\frac{1}{2}(3 j), & \text { if } x=u_{j} u_{j+1}, j \text { is even, } \\
5 p+7 q+5 m-\frac{1}{2}(3 j+1), & \text { if } x=v_{j} v_{j+1}, j \text { is odd, } \\
5 p+7 q+5 m-\frac{3}{2} j, & \text { if } x=u_{j} v_{j}, j \text { is even, } \\
2 p+4 q+\gamma_{3}-2 t & \text { if } x=z_{t} x_{t}, 1 \leq t \leq q, \\
5 p+7 q+\gamma_{4}-2 t, & \text { if } x=z_{t} y_{t}, 1 \leq t \leq q, \\
2 p+4 q+\gamma_{5}-2 t, & \text { if } x=x_{t} z_{t+1}, 1 \leq t \leq q, \\
5 p+7 q+\gamma_{6}-2 t, & \text { if } x=y_{t} z_{t+1}, 1 \leq t \leq q,\end{cases}
\end{aligned}
$$

where $\gamma_{1}=(1 / 2)(\lambda-1)(7 m-2)-(1 / 2)(\lambda-2)(7 m-1), \gamma_{2}=$ $(1 / 2)(\lambda-1)(3 m)-(1 / 2)(\lambda-2)(3 m-1), \gamma_{3}=(1 / 2)(\lambda-1)(3 m+$ $4)-(1 / 2)(\lambda-2)(3 m+3), \gamma_{4}=(1 / 2)(\lambda-1)(7 m+2)-(1 / 2)(\lambda-$ $2)(7 m+3), \gamma_{5}=(1 / 2)(\lambda-1)(3 m+2)-(1 / 2)(\lambda-2)(3 m+1)$, and $\gamma_{6}=(1 / 2)(\lambda-1)(7 m)-(1 / 2)(\lambda-2)(7 m+1)$. It is easy to verify that, for every edge $x y \in E\left(F_{1}\right), f(x)+f(x y)+f(y)=$ $8(p+q)+6 m$.

(b) We define $F_{2}$ as graph with $V\left(F_{2}\right)=V\left(F_{1}\right)$, where $c_{p+1}=v_{1}$ and $v_{m}=z_{1}$, and $E\left(F_{2}\right)=E\left(F_{1}\right)$. Under this definition, the cut vertices of $F_{2}$ are $c_{i}, 2 \leq i \leq p+1$, and $z_{t}, 1 \leq t \leq q$. Next, we define a bijection $f_{2}: V\left(F_{2}\right) \cup E\left(F_{2}\right) \rightarrow$ $\{1,2,3, \ldots, 7(p+q)+5 m-2\}$, where $f_{2}(x)=f_{1}(x)$ for all $x \in V\left(F_{2}\right) \cup E\left(F_{2}\right)$. It can be checked that $f_{2}$ is an edge-magic labeling of $F_{2}$ with the magic constant $8(p+q)+6 m$.

Open Problem 3. Let $p$ and $q \geq 1$ be integers.

(a) If $m \geq 3$ is an odd integer, find the super edge-magic deficiency of $C\left[C_{4}^{(p)}, L_{m}, c_{4}^{(q)}\right]$ with string $\left(2^{(p-1)}\right.$, $\left.m, 2^{(q-1)}\right)$

(b) If $m \geq 2$ is an even integer, find the super edge-magic deficiency of $C\left[C_{4}^{(p)}, L_{m}, c_{4}^{(q)}\right]$ with string $\left(2^{(p-1)}, m-\right.$ $\left.1,2^{(q-1)}\right)$. 
Theorem 8. Let $p, q \geq 2$ and $r \geq 1$ be integers.

(a) If $m \geq 2$ is an even integer and $H_{1}=C\left[C_{4}^{(p+q)}, L_{m}, c_{4}^{(r)}\right]$ with string $\left(2^{(p-2)}, 1^{(2)}, 2^{(q-1)}, m, 2^{(r-1)}\right)$, then $\mu\left(H_{1}\right)=0$.

(b) If $m \geq 3$ is an odd integer and $H_{2}=C\left[C_{4}^{(p+q)}, L_{m}, c_{4}^{(r)}\right]$ with string $\left(2^{(p-2)}, 1^{(2)}, 2^{(q-1)}, m-1,2^{(r-1)}\right)$, then $\mu\left(H_{2}\right)=0$.

Proof. (a) First, we define $H_{1}$ as a graph with $V\left(H_{1}\right)=\left\{a_{i}: 1 \leq\right.$ $i \leq 2 p\} \cup\left\{b_{i}: 1 \leq i \leq p+1\right\} \cup\left\{u_{j}: 1 \leq j \leq 2 q\right\} \cup$ $\left\{v_{j}: 1 \leq j \leq q+1\right\} \cup\left\{w_{s}: 1 \leq s \leq 2 m\right\} \cup\left\{x_{t}: 1 \leq t \leq\right.$ $2 r\} \cup\left\{y_{t}: 1 \leq t \leq r+1\right\}$, where $a_{2 p}=u_{1}, v_{q+1}=w_{1}$, and $w_{2 m}=y_{1}$, and $E\left(H_{1}\right)=\left\{b_{i} a_{i}, b_{i} a_{p+i}, a_{i} b_{i+1}, a_{p+i} b_{i+1}: 1 \leq\right.$ $i \leq p\} \cup\left\{v_{j} u_{j}, v_{j} u_{q+j}, u_{j} v_{j+1}, u_{q+j} v_{j+1} \mid 1 \leq j \leq q\right\} \cup$ $\left\{w_{s} w_{s+1}, w_{m+s} w_{m+s+1}: 1 \leq s \leq m-1\right\} \cup\left\{w_{s} w_{m+s}: 1 \leq s \leq\right.$ $m\} \cup\left\{y_{t} x_{t}, y_{t} x_{r+t}, x_{t} y_{t+1}, x_{r+t} y_{t+1}: 1 \leq t \leq r\right\}$.

Next, define a bijection $g_{1}: V\left(H_{1}\right) \cup E\left(H_{1}\right) \rightarrow\{1,2$, $3, \ldots, 7(p+q+r)+5 m-2\}$ as follows:

$$
\begin{aligned}
& g_{1}(z)= \begin{cases}6 p+7(q+r)+5 m+i-2, & \text { if } z=a_{i}, 1 \leq i \leq p, \\
3 p+q+r+m+1+i, & \text { if } z=a_{p+i}, 1 \leq i \leq p, \\
i, & \text { if } z=b_{i}, 1 \leq i \leq p+1, \\
4 p+q+r+m+j, & \text { if } z=u_{j}, 1 \leq j \leq q, \\
4(p+q+r)+3 m+j-1, & \text { if } z=u_{q+j}, 1 \leq j \leq q, \\
p+1+j, & \text { if } z=v_{j}, 1 \leq j \leq q+1, \\
p+q+1+s, & \text { if } z=w_{s}, s \text { is odd, } \\
4 p+2 q+r+m+\frac{1}{2} s, & \text { if } z=w_{s}, s \text { is even, } \\
4 p+5 q+4 r+3 m+\frac{1}{2}(s-1), & \text { if } z=w_{m+s}, s \text { is odd, } \\
p+q+1+s, & \text { if } z=w_{m+s}, s \text { is even, }\end{cases}
\end{aligned}
$$

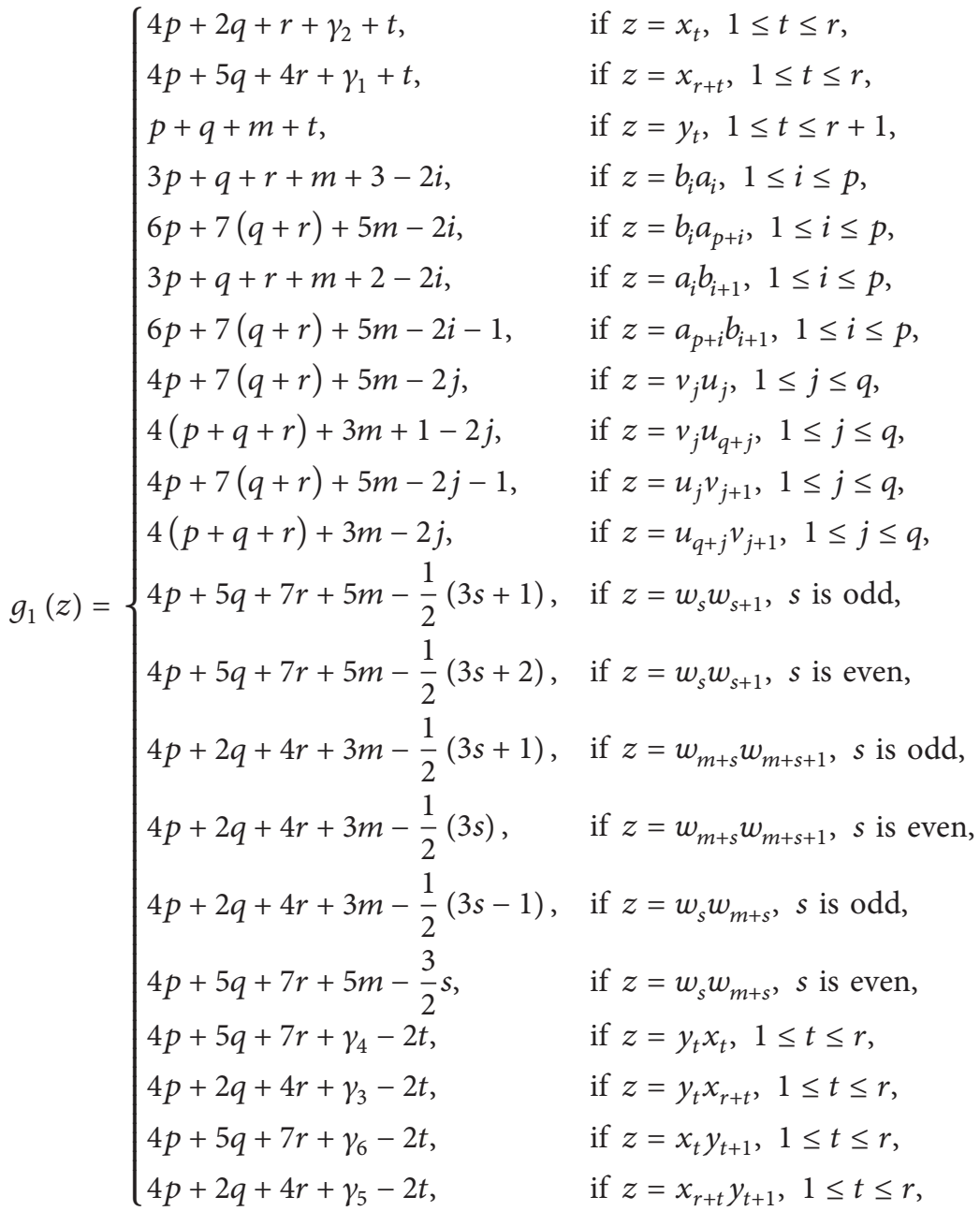


where $\gamma_{1}, \gamma_{2}, \gamma_{3}, \gamma_{4}, \gamma_{5}, \gamma_{6}$, and $\lambda$ are defined as in the proof of Theorem 7. It can be checked that, for every edge $x y \in E\left(H_{1}\right)$, $g_{1}(x)+g_{1}(x y)+g_{1}(y)=9 p+8(q+r)+6 m+1$. Hence $\mu\left(H_{1}\right)=0$.

An illustration of the labeling defined in the proof of Theorem 8 is given in Figure 1(b).

(b) We define $H_{2}$ as graph with $V\left(H_{2}\right)=V\left(H_{1}\right)$, where $a_{2 p}=u_{1}, v_{q+1}=w_{1}$, and $w_{m}=y_{1}$, and $E\left(H_{2}\right)=E\left(H_{1}\right)$. It can be checked that $g_{2}: V\left(H_{2}\right) \cup E\left(H_{2}\right) \rightarrow\{1,2,3, \ldots, 7(p+q+r)+$ $5 m-2\}$ defined by $g_{2}(x)=g_{1}(x)$, for all $x \in V\left(H_{2}\right) \cup E\left(H_{2}\right)$, is an edge-magic labeling of $H_{2}$ with the magic constant $9 p+$ $8(q+r)+6 m+1$.

Open Problem 4. Let $p, q \geq 2$ and $r \geq 1$ be integers.

(a) If $m \geq 3$ is an odd integer, find the edge-magic deficiency of $C\left[C_{4}^{(p)}, c_{4}^{(q)}, L_{m}, c_{4}^{(r)}\right]$ with string $\left(2^{(p-2)}, 1^{(2)}\right.$, $\left.2^{(q-1)}, m, 2^{(r-1)}\right)$.

(b) If $m \geq 2$ is an even integer, find the edge-magic deficiency of $C\left[C_{4}^{(p)}, c_{4}^{(q)}, L_{m}, c_{4}^{(r)}\right]$ with string $\left(2^{(p-2)}, 1^{(2)}\right.$, $\left.2^{(q-1)}, m-1,2^{(r-1)}\right)$.

\section{Conflicts of Interest}

The authors declare that there are no conflicts of interest regarding the publication of this paper.

\section{Acknowledgments}

The first author has been supported by "Hibah Kompetensi 2016” (018/SP2H/P/K7/KM/2016) from the Directorate General of Higher Education, Indonesia.

\section{References}

[1] A. Kotzig and A. Rosa, "Magic valuations of finite graphs," Canadian Mathematical Bulletin, vol. 13, pp. 451-461, 1970.

[2] H. Enomoto, A. S. Llado, T. Nakamigawa, and G. Ringel, "Super edge-magic graphs," SUT Journal of Mathematics, vol. 34, no. 2, pp. 105-109, 1998.

[3] R. M. Figueroa-Centeno, R. Ichishima, and F. A. MuntanerBatle, "On the super edge-magic deficiency of graphs," Electronic Notes in Discrete Mathematics, vol. 11, pp. 299-314, 2002.

[4] A. A. Ngurah, E. T. Baskoro, and R. Simanjuntak, "On super edge-magic deficiency of graphs," Australasian Journal of Combinatorics, vol. 40, pp. 3-14, 2008.

[5] J. A. Gallian, “A dinamic survey of graph labelings," Electron. J. Combin, vol. 16, 2015, DS6.

[6] R. M. Figueroa-Centeno, R. Ichishima, and F. A. MuntanerBatle, "The place of super edge-magic labelings among other classes of labelings," Discrete Mathematics, vol. 231, no. 1-3, pp. 153-168, 2001. 


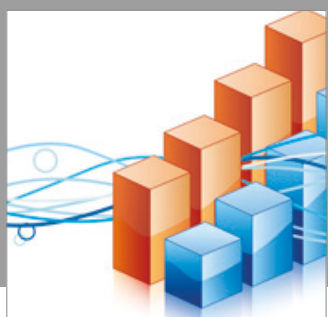

Advances in

Operations Research

vatersals

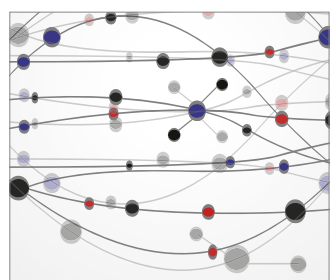

\section{The Scientific} World Journal
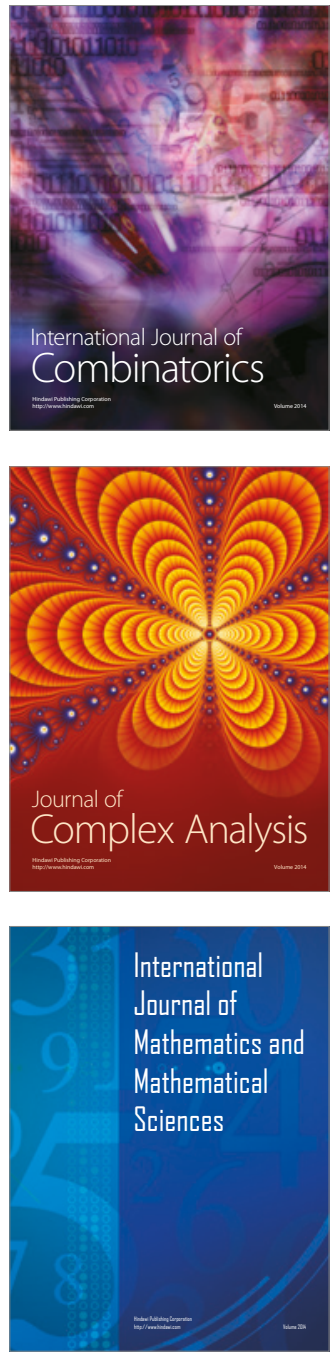
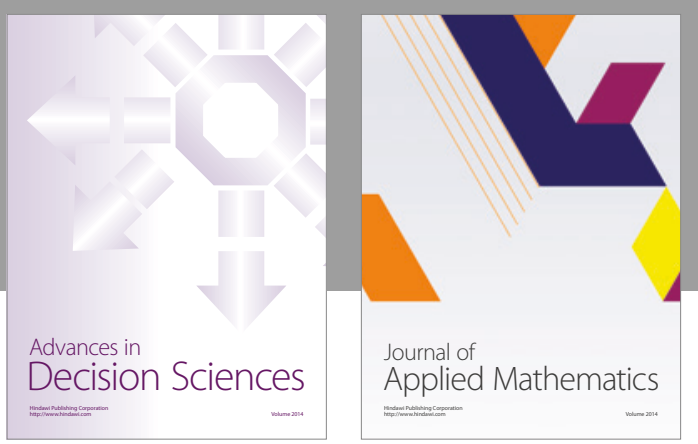

Algebra

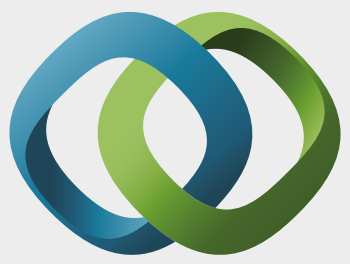

\section{Hindawi}

Submit your manuscripts at

https://www.hindawi.com
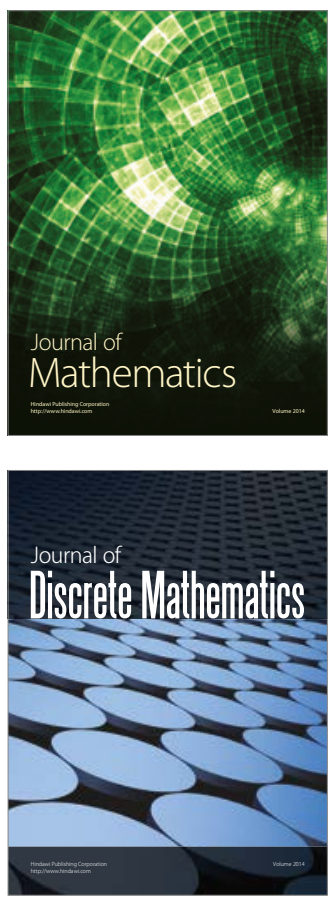

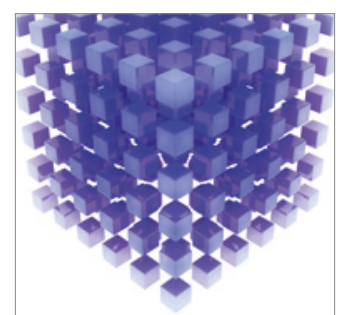

Mathematical Problems in Engineering
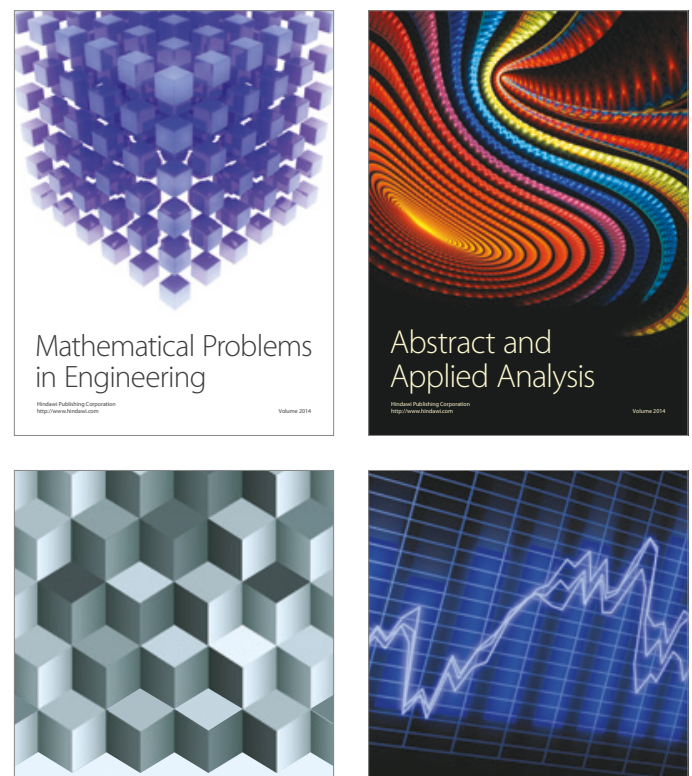

Journal of

Function Spaces

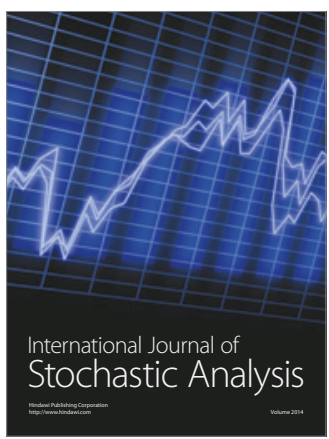

Probability and Statistics
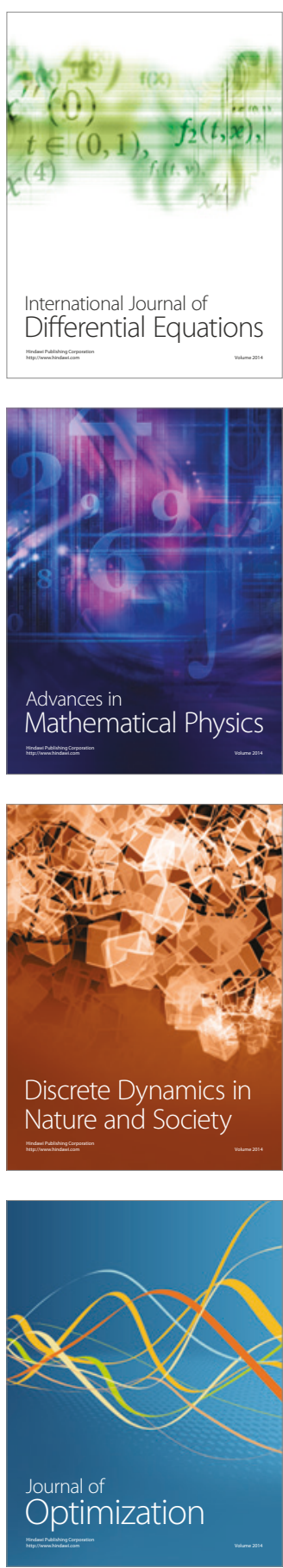